\title{
Traduire
}

Une autre perspective sur r tr traduction

Revue française de la traduction

$243 \mid 2020$

Les arts du spectacle

\section{Joining le cirque}

\section{Clíona Ní Ríordáin}

\section{(2) OpenEdition}

\section{Journals}

Édition électronique

URL : http://journals.openedition.org/traduire/2228

DOI : 10.4000/traduire.2228

ISSN : 2272-9992

\section{Éditeur}

Société française des traducteurs

\section{Édition imprimée}

Date de publication : 15 décembre 2020

Pagination : 119-127

ISSN : 0395-773X

\section{Référence électronique}

Clíona Ní Ríordáin, « Joining le cirque », Traduire [En ligne], 243| 2020, mis en ligne le 15 décembre 2020, consulté le 31 décembre 2020. URL : http://journals.openedition.org/traduire/2228; DOI : https://doi.org/10.4000/traduire.2228 


\section{Joining le cirque}

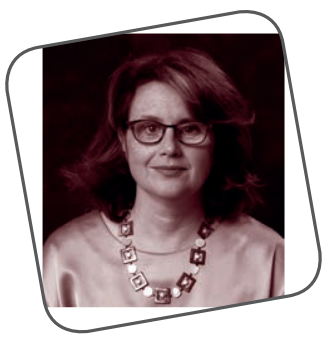

\section{Clíona Ní Ríordáin}

La traduction en anglais de la pièce de cirque HUMAN (articulations) de Christophe Huysman a été une véritable aventure traductive, qui a commencé par un échange entre ma collègue Isabelle Perrin et Cyril Thomas, responsable recherche et développement au Centre national des arts du cirque (CNAC). La commande était destinée à une publication en France à l'occasion du $32^{e}$ Festival Circa d'Auch. Outre le texte de la pièce, Cyril Thomas m'a transmis une version filmée du spectacle, réalisée en mars 2019 par la $32^{e}$ promotion du CNAC. Cette version filmée constituait un élément essentiel, car elle m'a aidée à saisir la jubilation corporelle, qui fait partie intégrante de la pièce.

Christophe Huysman souligne dans l'avant-propos de la pièce que le texte est né d'une expérimentation sur les techniques du cirque. Gérard Fasoli (scénographe de la pièce et actuel directeur du CNAC) en raconte la genèse dans ce même document introductif, afin que le lecteur comprenne que les arts du cirque sont à l'origine de la création de Christophe Huysman:

Il y avait des spécialités cirque: le cadre, pour Antoine et Colline, le mât, pour William. Le mât pouvait à la fois être un support de la structure et un support de technique de cirque. La façon dont on traite le mât est d'un niveau technique basique, accessible, pour un artiste circassien bien préparé physiquement, en trois mois de répétition. C'est comme ca que sont arrivés les trois 
mâts. Les aiguilles, au départ, devaient être une table, un plan qui pivotait à l'horizontale: l'idée était de donner à Christophe un semblant de scène, et à Manu un endroit où il pourrait s'exprimer - il est acrobate-équilibriste. Et puis, comme le texte a beaucoup développé la notion de temps, et d'urgence, l'idée des aiguilles s'est imposée. Florent est fil de ferriste: tendre un fil est très contraignant, les salles de théâtre ne le permettent pas la plupart du temps.

On saisit d'emblée l'importance des éléments concrets dans l'élaboration de la pièce, et la spécificité du monde circassien. Des termes comme "mât», par exemple, ont une signification bien particulière dans la culture circassienne. On ne traduit pas ce terme par mast, qui renvoie au vocabulaire de la marine, mais par pole. De même, plus loin dans le descriptif, Florent est qualifié de «fil de ferriste»: ce terme, également orthographié «fil de fériste», se traduit par tightrope acrobat, et dans le cas d'un funambule, on dira highwire acrobat. Un autre terme, aerialist, est utilisé pour les artistes comme Antoine et Colline, qui font du cadre aérien, ou pour les artistes à la corde lisse (corde verticale). À la lecture de ces descriptions liminaires, qui marquaient mon initiation à un nouvel univers, j'ai été saisie du désir de faire un stage de cirque, ou, dans les termes déterminés par la langue anglaise, d'une folle envie de m'enfuir comme le ferait un vagabond romantique, to join the circus.

L'été que j'ai passé en compagnie de la pièce s'est mué de facto en une sorte de fugue traductive. Mon voyage au pays du cirque a été nourri par la lecture d'articles critiques en anglais sur les arts du cirque dans le journal Popular Entertainment Studies. Cela m'a permis, par exemple, de comprendre l'importance de ce domaine en Argentine à la fin $d u X x^{e}$ siècle, et l'évolution du cirque contemporain, qui a abandonné le three-ring circus, marqué par la présence d'animaux, au profit d'une exécution performative. Pour me familiariser avec les pratiques et les institutions du cirque en langue anglaise, j'ai parcouru les sites internet des équivalents du CNAC dans l'anglosphère: le National Institute of Circus Arts en Australie, qui propose un Bachelor of Circus Arts, et son école pour jeunes enfants Flying Fruit Fly Circus [le cirque des moucherons à fruits], ou encore le National Centre for Circus Arts à Londres. Cette immersion 
dans le monde très singulier du cirque m'a permis de constituer une base terminologique autour du sujet. Au-delà d'une recherche langagière, j'ai pu, pendant le temps de la traduction, vivre en Circassie, ce qui est pour moi l'un des aspects les plus délicieux du métier de traducteur. En effet, notre travail nous permet de nous évader, d'habiter la peau d'un autre pendant le temps dilaté du traduire.

Cependant, le texte de Christophe Huysman fait également appel à un vocabulaire qui dépasse celui du cirque. Profondément politique, il se caractérise par une rage contre l'injustice et une empathie avec ceux qui ont été humiliés et qui «ont perdu leur voix, leur voie», pour citer le programme du Festival Circa à Auch en 2006. Huysman donne à voir un monde déshumanisé: dans la partie de la pièce intitulée "Perdus 2 », il recourt au vocabulaire de la culture managériale pour expliquer l'effet réel de la novlangue sur les interactions humaines. S'appuyant sur des expressions comme "objectifs à atteindre», "prendre en compte des paramètres», il déploie la terminologie des allocations, avec son lot de sigles, et aligne les catégorisations déployées par Pôle emploi pour que le spectateur comprenne l'aliénation inhérente à ce système. Par son écriture serrée, Huysman multiplie les références, comme "allocation spécifique de solidarité», "allocation logement» ou encore "revenu minimum d'insertion». Le monologue qui s'ensuit nous fait comprendre la frustration qui domine les échanges avec les institutions:

bonjour je réponds à l'annonce merci de laisser votre message ou de rappeler ultérieurement

veuillez laisser un message votre correspondant vous joindra dès son retour

allô oui bonjour est-ce que je pourrais parler

vous n'avez pas le numéro là?

tut tut tut

allô allô bonjour monsieur c'est pour avoir un rendez-vous d'accord, donc au 33 rue de l'Avenir c'est une femme merci beaucoup au revoir ca j'annule et l'assistante sociale j'annule des questions comme ca qui est là y a qui y a qui y a personne (les mots disparaissent peu à peu) 
Hello l'm answering your ad please leave your message or call back later

Please leave a message the person you have called will ring you back on their return

Hello yes good day could I speak to

Don't you have that number?

Tut tut tut

Hello hello good day sir I want to make an appointment

Yes at 33 rue de l'Avenir it's a woman thank you very much goodbye

I'm cancelling that and I'm cancelling the social worker

Questions like who is there who's there no one's there

(the words are disappearing bit by bit)

Nous voilà dans un autre monde, beaucoup moins jubilatoire. Le vocabulaire utilisé ici par Huysman correspond à une réalité sociale proprement française. Le dramaturge lui-même est conscient de l'évolution de cette terminologie, car il précise la mention "actualiser terminologies et allocations selon». Ici, de nouveau, le traducteur est interpellé. Il doit, encore une fois, s'armer de compétences techniques, mais plutôt que de s'appuyer sur une base terminologique, il lui faut choisir à quelle réalité anglophone (anglaise, américaine, irlandaise?) il va faire correspondre le tissu social français. Ce domaine pose les mêmes problèmes que le domaine juridique: le droit français ne peut se «translater»vers le common law anglais, nous nous trouvons face à une réalité extralangagière. Par ailleurs, les choix terminologiques ont un effet domino sur l'ensemble des références culturelles qui seront employées tout au long de la pièce. J'ai choisi d'adopter le vocabulaire du système britannique, notamment en raison de titres comme Queens Counsel (QC) qui ancrent bien le texte dans la réalité de la langue anglaise - davantage en tout cas qu'un terme plus passe-partout, comme lawyer. Ainsi, dans la traduction du passage suivant, la terminologie anglaise est perceptible. Le lecteur attentif notera toutefois que je n'ai pas opté pour une conversion de l'argent en livres sterling; puisque la traduction était destinée à une publication en France, il aurait été superflu, dans ces conditions, de proposer une conversion monétaire. On pourrait envisager la chose si la pièce se jouait hors de France. 
Pour mai mon ASS arrivant à terme il est primordial pour moi de développer rapidement mon activité afin de pouvoir m'assurer un minimum de rémunération rémunération qui devra être supérieure ou égale à mon ASS soit $434 €$ (actualiser terminologies et allocations selon). Il faudra toutefois faire attention car mon exonération de charges sociales arrive à terme elle en juin. Pour septembre-octobre j'espère pouvoir me sortir définitivement de ma précarité en m'octroyant au minimum un Smic voire plus si mon activité peut me le permettre sinon il me faudra envisager de constituer un dossier RMI si toutefois la possibilité m'en est offerte car je ne suis qu'un homme.

As my income support is going to be cut in May I really need to develop my activity so that I can earn a minimum revenue a revenue that should be equal to or more than my income support of $€ 434$ (figures and terms can be changed and brought up to date). However, I have to be careful because I will no longer be exempt from social security contributions from the end of June. For Septemberl October I hope to put an end to my precarious financial situation permanently by making at least the minimum wage every month, otherwise l'll have to see if I can put together a dossier to get emergency benefit if I can do so because I am only a man.

Plus loin dans la pièce, ce choix traductif influe sur le passage évoquant le besoin de recourir à un avocat: "Je recherche maittre Dérouillé» devient l'm looking for Mr Hammered QC.

Huysman, comme le montre la citation précédente, utilise des noms propres à valeur sémantique. Outre «maître Dérouillé », il est également question de Monsieur Toutou et de la famille Toutou. En anglais, ils deviennent Mr Doggy et the Doggy family; par extension, certaines répliques de la conversation téléphonique sont traduites par bow-wow, l'onomatopée associée en anglais aux aboiements d'un chien. Et comme la pièce regorge de jeux de mots parfois difficiles à traduire, l'introduction de ce réseau sémantique permet de proposer des solutions compensatoires telles que l'm going to the dogs à la place de "Je suis à vau-l'eau», afin de faire en sorte que la texture langagière de la pièce dans la langue cible contienne autant de joutes verbales que le texte source. 
L'attrait du texte de Christophe Huysman vient aussi de son inscription dans une généalogie littéraire qui remonte à Samuel Beckett. Dans HUMAN, le corps et les provesses physiques trouvent un écho dans l'agilité verbale de l'auteur, ramenant le spectateur au slapstick. Beckett adorait cette tradition de la comédie bouffonne physique du film muet qui puise ses racines dans le music-hall et que l'on retrouve dans les films de Charlie Chaplin ou de Buster Keaton. Ce même Buster Keaton joua d'ailleurs dans Film, le long métrage expérimental de Samuel Beckett réalisé par Alan Schneider en 1965. En s'appuyant sur cette tradition, la pièce HUMAN débute par une injonction physique:

- J'ai buté j'ai buté Buté d'dans buté Là Juste là J'ai buté à c't'endroit

- Tu penses ce que tu dis?

- J'y réfléchis

- Tu penses ce que tu dis?

- J'y réfléchis j'y réfléchis Là j'y réfléchis encore je me sens bleu

- Tu penses

- Encore?

On note l'absence de point, et la génération du texte par l'action corporelle.

- I butted, I butted Butt into the butt There Just there in that spot

-Are you thinking about what are you saying?

- I'm giving it some thought

-Are you thinking about what are you saying?

-l'm giving it some thought, some thought, there I'm giving it some thought again and I feel blue

-You are thinking

-Still?

Ailleurs, comme dans un texte de Beckett, ce sont les sonorités qui circulent et nourrissent la construction textuelle:

J'ai un grand cœur un cœur qui bat pas bas mon cœur pourquoi?

Qui bat pas bas qui bat 
Hi Ha Ho Hé

- Hé Ho

- Ha Hi Hé HO

- Hé Ho

- Ha Un grand coeur j'en ai jusque-là!

Et j'ai aussi des pieds des pieds? Des petits pieds des petits pieds et des grands pieds et du pia pia tarses métatarses tendons tiens donc!

Mais qui m'a fait mais qui? mais qui m'a fait ce que je suis?

Ah là Ha fo fo faudra ca pour tenir droit du col au cul je tiens dessus

Je tiens dessus!

Hé HO Ha faudrait pas qu'à force de tenir tout ca le cul se colle au col et le col descend déçu et et et HO d'un coup mon cou crâne et

croule sous le coup du cul qui colle au sol déçu J'en sue J'en sue du col

On perçoit le lien entre l'énonciation syllabique et le mouvement du corps. Pour traduire cette section de la pièce, ce sont les nursery rhymes (comptines) et les nonsense rhymes d'Edward Lear qui ont guidé mes choix traductifs. À cela s'ajoute l'importance de réciter à voix haute les segments de la traduction afin de trouver les bons appuis.

I have a big heart that is beating pit a pat why my heart why? Which beats pit-pat

$\mathrm{HaHi}$ Heigh $\mathrm{HO}$

- Heigh Ho

- Heigh Ho Heigh $\mathrm{HO}$

- Heigh Ho

- Ha I have a big heart, so big it goes right down to the ground! And I also have feet feet. Little feet little feet and

big feet and ta ta tarsus metatarsus tendons tendencies!

But who made but who? But who made me what I am?

And there Hah have have to have that to hang on from the top to the bottom

I'm sitting on it

I'm sitting on it!

Heigh HO hah can't have it said that having been sitting on it the bottom bastes to the top and the top descends disappointed 
and and and HO then my top tenses and topples over tension of the bottom which is basted disappointed to the floor. I' $m$ sweating I'm sweating from my disappointed to

Dans cet extrait, nous remarquons l'importance du rythme. Les énonciations scandées par une variation de voyelles «Ha $\mathrm{Hi} H \mathrm{H} \mathrm{HO}$ », qui deviennent en anglais $\mathrm{Ha} \mathrm{Hi} \mathrm{Heigh} \mathrm{HO}$, ressemblent à des vocalises, et cette flexibilité vocale déclenche une quête existentielle: "Mais qui m'a fait mais qui? mais qui m'a fait ce que je suis?" La réponse générée par cette question prend la forme d'une démonstration d'agilité physique et verbale, où la dextérité des circassiens trouve son écho dans le texte, dominé par des jeux de mots. Cette modulation n'est pas reproduite dans la traduction, faute de monosyllabes qui refléteraient les sonorités du texte source. Puisque la commande prévoyait une traduction pour l'édition et non pour la scène, j'ai décidé de privilégier le sens et reproduit les jeux de mots, consciente que ce choix serait au détriment du rythme. Encore une fois, la fonction attribuée au texte traduit a influencé le processus traductif: une traduction destinée à être jouée ferait certainement abstraction du sens pour produire un texte plus riche en termes rythmiques.

Cette traduction a été nourrie de nombreux échanges avec Christophe Huysman et Cyril Thomas. Nos conversations et nos courriels ont donné à "cet été au cirque»la dimension collaborative qui caractérise le travail avec le monde du spectacle vivant. Tout au long de la pièce, Huysman se met au service des arts du cirque en proposant un texte qui valorise et vient en appui aux compétences des artistes circassiens. En cela, son écriture, dans le cadre fourni par le CNAC, relève d'un art voisin de celui des traducteurs: nous utilisons les contraintes imposées, nous les exploitons pour en faire une force créative, jouant ainsi à notre tour aux équilibristes, conjuguant, comme le dit si bien Christophe Huysman, "un acte grammatical complexe avec autant de disciplines que de présences sur le plateau». C'est tout cela qui a fait le bonheur de traduire cette pièce circassienne, art emblématique du spectacle vivant.

cliona.ni-riordain@sorbonne-nouvelle.fr 
Clíona Ní Ríordáin est professeure des universités à la Sorbonne Nouvelle où elle enseigne la traduction et la littérature irlandaise et fait partie de l'équipe TRACT au sein de l'unité de recherche PRISMES EA4398. Traductrice, notamment de Cavalier passe ton chemin (Michel Déon), chez Lilliput Press, elle est l'auteure de trois anthologies de poésie irlandaise, la dernière en date, Jeune Poésie d'Irlande: les poètes du Munster (IIlador, 2015), codirigée et cotraduite avec Paul Bensimon. Elle est également fondatrice et présidente du prix de traduction de la Fondation irlandaise, attribué pour la première fois en 2019 à Mona de Pracontal pour sa traduction de Rien d'autre sur terre de Conor O'Callaghan. 